\title{
Metastable States of Quantum Lattice Systems
}

\author{
Geoffrey L. Sewell \\ Department of Physics, Queen Mary College, London E1 4NS, England
}

\begin{abstract}
We extend the characterisation of metastability, as given in [1] for classical systems, to show that quantal lattice systems with suitable long range forces can support metastable states.
\end{abstract}

In a previous work [1], we characterised metastable states of classical systems as being locally, but not globally, thermodynamically stable and also stable under constraints confining the system to a suitable reduced state space. It was shown that, according to this characterisation (whose motivation and relation to other ones $[2,3]$ was discussed in [1]), systems with certain types of long range forces can support metastable states. The object of this note is to show that similar conclusions are applicable to quantal lattice systems (cf. the Proposition and following Comment below).

Our treatment is based on a definition of local thermodynamical stability of such systems, that was introduced in [4]. There it was shown that, for systems with short range forces, the conditions for local stability are equivalent to those of KMS. In the present note, we need to extend our definition of local stability to systems with long range forces.

We formulate the states, observables and forces of a system on a lattice, $\Gamma\left(=Z^{v}\right.$, say) in a standard way (cf. [5]). Thus, denoting the set $\{\Lambda\}$ of finite point subsets of $\Gamma$ by $L$, we define the $C^{*}$-algebra $\mathscr{A}$ of observables of the system as that generated by a family $\{\mathscr{A}(\Lambda) \mid \Lambda \in L\}$ of local $C^{*}$-algebras, where $\mathscr{A}(\Lambda)$ is a type- $I$ factor of finite order that is isotonic with respect to $\Lambda$. The state space $\Omega$ is then the set of positive normalised linear functionals on $\mathscr{A}$. The set of translationally invariant elements of $\Omega$ will be denoted by $\bar{\Omega}$. The forces in the system will be taken to correspond to an interaction potential $\phi$, which maps $L$ into the self-adjoint elements of $\mathscr{A}$ in such a way that (i) $\phi(\emptyset)=0$; (ii) $\phi(\Lambda) \in \mathscr{A}(\Lambda)$; (iii) $\phi$ transforms covariantly w.r.t. space translations; and (iv)

$$
|\phi| \equiv \sum_{0 \in \Lambda} \frac{\|\phi(\Lambda)\|}{N(\Lambda)}<\infty
$$


where $N(\Lambda)$ is the number of points in $\Lambda$. We define $\Phi$ to be the Banach space of such interactions, with norm $|\cdot|$; and we define $\Phi_{1}(\subset \Phi)$ to be the Banach space of those interactions $\phi$ for which the norm $|\phi|_{1}<\infty$, where

$$
|\phi|_{1} \equiv \sum_{0 \in \Lambda}\|\phi(\Lambda)\| .
$$

For $\Lambda \in L$ and $\phi \in \Phi$, we define the local energy functional $E_{\Lambda}$ on $\Omega$ by the formula

$$
E_{\Lambda}(\omega)=\sum_{\Lambda^{\prime} \subset \Lambda} \omega\left(\phi\left(\Lambda^{\prime}\right)\right) \forall \omega \in \Omega .
$$

Let $\tilde{\Omega}$ be the set of states $\omega$ such that, for any state $\omega^{\prime}$ which coincides with $\omega$ outside some bounded region of $\Gamma, E_{\Lambda^{\prime}}\left(\omega^{\prime}\right)-E_{\Lambda^{\prime}}(\omega)$ converges, as $\Lambda^{\prime} \rightarrow \infty$, to a value in $R \cup\{\infty\}$ that does not depend on the particular chosen sequence of $\Lambda^{\prime \prime}$ s. Thus $\tilde{\Omega}=\Omega$ if $\phi \in \Phi_{1}$, though not, in general, if $\phi \in \Phi \backslash \Phi_{1}$. For $\omega \in \tilde{\Omega}$ and $\omega^{\prime} \in \Omega$, with $\omega_{\Lambda_{c}}^{\prime}=\omega_{\Lambda_{c}}$, where $\Lambda_{c}=\Gamma \backslash \Lambda$, we define

$$
\Delta \tilde{E}_{\Lambda}\left(\omega^{\prime} \mid \omega\right)=\lim _{\Lambda^{\prime} \uparrow}\left[E_{\Lambda^{\prime}}\left(\omega^{\prime}\right)-E_{\Lambda^{\prime}}(\omega)\right] .
$$

Let $S_{A}, \tilde{S}_{A}$ be the local entropy and conditional local entropy functionals on the state space, as defined in [4]. We define the free energy density functional $f$ on $\bar{\Omega}$, for temperature $T$, by the formula

$$
f(\omega)=\lim _{\Lambda \uparrow}\left[E_{\Lambda}(\omega)-T S_{\Lambda}(\omega)\right] / N(\Lambda) ;
$$

and, for $\omega \in \tilde{\Omega}$ and $\omega_{\Lambda_{c}}^{\prime}=\omega_{\Lambda_{c}}$, we define the incremental local conditional free energy $\Delta \tilde{F}_{\Lambda}\left(\omega^{\prime} \mid \omega\right)$ by the formula

$$
\Delta \tilde{F}_{\Lambda}\left(\omega^{\prime} \mid \omega\right)=\Delta \tilde{E}_{\Lambda}\left(\omega^{\prime} \mid \omega\right)-T\left(\tilde{S}_{\Lambda}\left(\omega^{\prime}\right)-\tilde{S}_{\Lambda}(\omega)\right) .
$$

In cases where $\phi \in \Phi_{1}, \Delta \tilde{F}_{\Lambda}\left(\omega^{\prime} \mid \omega\right)=\tilde{F}_{\Lambda}\left(\omega^{\prime}\right)-\tilde{F}_{\Lambda}(\omega)$, where $\tilde{F}_{\Lambda}$ is the conditional free energy functional of [4].

The following definition provides the quantal analogues of those given in [1] for local and global stability, equilibrium and metastability.

Definition 1. (i) We define $K_{L}$, the set of locally thermodynamically stable states of the system, to be those elements of $\tilde{\Omega}$ for which $\Delta \tilde{F}_{\Lambda}\left(\omega^{\prime} \mid \omega\right) \geqq 0 \forall \Lambda \in L, \omega_{\Lambda_{c}}^{\prime}=\omega_{\Lambda_{c}}$.

(ii) Let $\Omega_{0}$ be a closed proper subset of $\Omega$. We define $\bar{K}_{G}$ (resp. $\bar{K}_{0}$ ), the set of globally stable (resp. $\Omega_{0}$-stable) translationally invariant states of the system to be the set of elements of $\bar{\Omega}$ (resp. $\bar{\Omega}_{0} \equiv \Omega_{0} \cap \bar{\Omega}$ ) at which $f$ (resp. $f_{\mid \Omega_{0}}$ ) attains its lower bound.

(iii) We define $\bar{K}_{E}$, the set of translationally invariant equilibrium states of the system, to be $\bar{K}_{G} \cap K_{L}$.

(iv) We define $\bar{K}_{M_{0}}$, the set of $\Omega_{0}$-metastable, translationally invariant states of the system, to be $\bar{K}_{0} \cap K_{L} \backslash \bar{K}_{G}$.

It follows from these definitions and the proof of Part (a) of the theorem of [4(a)] that, if $\phi \in \Phi_{1}$, then $K_{L} \cap \bar{\Omega} \subset \bar{K}_{G}$; and therefore $K_{L} \cap \bar{\Omega}=\bar{K}_{E}$ and $\bar{K}_{M_{0}}=\emptyset$. On the other hand we shall presently construct a class of models, with $\phi \in \Phi \backslash \Phi_{1}$, for which $\bar{K}_{M_{0}} \neq \emptyset$. Thus we arrive at the following 
Proposition 1. (i) If $\phi \in \Phi_{1}$, then $K_{L} \cap \bar{\Omega}=\bar{K}_{E}$ and $\bar{K}_{M_{0}}=\emptyset$; and (ii) for suitable $\mathscr{A}$, $\phi\left(\in \Phi \mid \Phi_{1}\right), T$ and $\Omega_{0}, \bar{K}_{M_{0}} \neq \emptyset$.

Comment. This result parallels those of [1; Section 2]. Further, it may readily be seen that the result given by [1; Proposition 3.1] concerning metastable states in mean field theoretic classical models may be carried over to quantal lattice systems. Thus, models with suitable short range interactions together with long range mean field theoretic ones (defined in analogy with [1; Section 3] can support metastable states whose thermodynamic functions are real analytic continuations, in temperature and chemical potential, of those of an equilibrium phase. Similar results for mean field theoretic models have earlier been obtained on different bases $[2,6]$.

Models with Metastable States. We now formulate our specifications of $\mathscr{A}(\Lambda), \phi$, and $\Omega_{0}$ for the models, referred to before Proposition 1 , which will be shown to support metastable states. Thus we take $\mathscr{A}(\Lambda)$ to be a type-I factor of order $n^{N(\Lambda)}$, with $n<\infty$. Let $\left\{P_{\Lambda} \in \mathscr{A}(\Lambda) \mid \Lambda \in L\right\}$ be a family of projectors such that (i) $P_{\Lambda_{1}} P_{A_{2}}$ $=P_{\Lambda_{1} \cup \Lambda_{2}}$, (ii) $P_{\Lambda} \mathscr{A}(\Lambda) P_{\Lambda}$ is a type- $I$ factor of order $m^{N(\Lambda)}$, with $m<n$, and (iii) $P_{\Lambda}, \mathscr{A}(\Lambda)$ transform covariantly with $\Lambda$ under space translations. It may be verified that these properties are obtained if (a) $\mathscr{A}(\Lambda)$ is isomorphic with the set of bounded operators in a Hilbert space $\mathscr{H}_{A}$ of dimension $n^{N(\Lambda) / 2}$, such that $\mathscr{H}_{\Lambda_{1}} \otimes \mathscr{H}_{\Lambda_{2}} \approx \mathscr{H}_{\Lambda_{1} \cup \Lambda_{2}}$ for $\Lambda_{1} \cap \Lambda_{2}=\emptyset$; (b) $P_{\Lambda}$ corresponds to the projector from $\mathscr{H}_{\Lambda}$ to a subspace $\mathscr{K}_{\Lambda}$ of dimension $m^{N(\Lambda) / 2}$, with $\mathscr{K}_{\Lambda_{1}} \otimes \mathscr{K}_{\Lambda_{2}} \approx \mathscr{K}_{\Lambda_{1} \cup \Lambda_{2}}$ for $\Lambda_{1} \cap \Lambda_{2}=\emptyset$; and (c) $\mathscr{H}_{\Lambda}, \mathscr{K}_{\Lambda}$ transform covariantly with $\Lambda$ under space translations.

The interaction $\phi$ is assumed to be of the form $\phi_{1}+\phi_{2}$, where $\phi_{1} \in \Phi_{1}$, and $\phi_{2} \in \Phi \backslash \Phi_{2} ;$ and, for all $\Lambda \in L, \quad\left(1-P_{\Lambda}\right) \phi_{1}(\Lambda)=0$ and $\phi_{2}(\Lambda)=\left(I-P_{\Lambda}\right) \phi_{2}(\Lambda)$ $>c(\Lambda)\left(I-P_{\Lambda}\right)$ where $c(\Lambda)$ is a positive number and $\sum_{0 \in \Lambda} c(\Lambda)=\infty$.

We take $\Omega_{0}$ to be $\left\{\omega \in \Omega \mid \omega\left(I-P_{\Lambda}\right)=0 \forall \Lambda \in L\right\}$.

We now establish that the models of this class support $\Omega_{0}$-metastable states by showing that (a) $\bar{K}_{0} \subset K_{L}$ and (b) for sufficiently large $T, \bar{K}_{0} \cap \bar{K}_{G}=\emptyset$.

Proof of $(a)$. We start by introducing an auxiliary $C^{*}$-algebra $\mathscr{B}$ as follows. Let $\mathscr{B}(\Lambda)$ be the $W^{*}$-algebra $P_{\Lambda^{\prime}} \mathscr{A}(\Lambda) P_{\Lambda}$, whose unit element is $P_{\Lambda}$; and, for $\Lambda^{\prime} \supset \Lambda$, let $i_{\Lambda^{\prime} \Lambda}$ be the injective mapping of $\mathscr{B}(\Lambda)$ into $\mathscr{B}\left(\Lambda^{\prime}\right)$ given by: $i_{\Lambda^{\prime} \Lambda}\left(B_{\Lambda}\right)=P_{\Lambda^{\prime} \backslash \Lambda^{\prime}} B_{\Lambda^{\prime}} P_{\Lambda^{\prime} \backslash \Lambda}$. We identify $B_{\Lambda}(\in \mathscr{B}(\Lambda))$ with $i_{\Lambda^{\prime} \Lambda}\left(B_{\Lambda}\right)\left(\in \mathscr{B}\left(\Lambda^{\prime}\right)\right)$ for $\Lambda^{\prime} \supset \Lambda$, thereby rendering $\mathscr{B}(\Lambda)$ isotonic in $\Lambda$. We then define $\mathscr{B}$ to be the quasi-local $C^{*}$-algebra given by the norm closure of $\bigcup_{\Lambda \in L} \mathscr{B}(\Lambda)$.

It follows from our definitions of $\Omega_{0}$ and $\mathscr{B}$ that the elements $\omega$ of $\Omega_{0}$ are completely determined by their restrictions $\omega_{\mid \mathscr{B}(\Lambda)}$ to the local algebras $\mathscr{B}(\Lambda)$, and may thus be identified with the states on $\mathscr{B}$. Further, as $\left(I-P_{\Lambda}\right) \phi_{1}(\Lambda)=0$ and $P_{\Lambda} \phi_{2}(\Lambda)=0$, it follows that $\bar{K}_{0}$ corresponds to the set of globally stable states on $\mathscr{B}$ for the interaction $\phi_{1}$. Hence, applying Definition 1 (i) and Proposition 1 (i) to the states on $\mathscr{B}$ for interaction $\phi_{1}$, we see that $\Delta \tilde{F}_{\Lambda}\left(\omega^{\prime} \mid \omega\right) \geqq 0 \forall \Lambda \in L, \omega \in \bar{K}_{0}, \omega^{\prime} \in \Omega_{0}$ and $\omega_{\Lambda_{c}}^{\prime}=\omega_{\Lambda_{c}}$. Thus it remains for us to show that $\Delta \tilde{F}_{\Lambda}\left(\omega^{\prime} \mid \omega\right) \geqq 0$ if $\omega \in \bar{K}_{0}, \omega^{\prime} \in \Omega \backslash \Omega_{0}$ and $\omega_{\Lambda_{c}}^{\prime}=\omega_{\Lambda_{c}}$. This we shall do by proving that, for such states, $\Delta \tilde{E}_{\Lambda}\left(\omega^{\prime} \mid \omega\right)=\infty$ and therefore, as $\tilde{S}_{A}$ is a bounded functional on $\Omega$ [4(a); Lemma 2.4 (2)], $\Delta \tilde{F}_{A}\left(\omega^{\prime} \mid \omega\right)=\infty$.

Assuming then that $\omega \in \bar{K}_{0}, \omega^{\prime} \in \Omega \backslash \Omega_{0}$ and $\omega_{\Lambda_{c}}^{\prime}=\omega_{\Lambda_{c}}$, we start by showing that there exists a point $i$ in $\Lambda$ such that $\omega^{\prime}\left(P_{i}\right)<1$, where $P_{i} \equiv P_{\{i\}}$. For this purpose we 
start by assuming the contrary, namely that $\omega^{\prime}\left(P_{i}\right)=1 \forall i \in \Lambda$. This in turn implies that $\omega^{\prime}\left(P_{i}\right)=1 \forall i \in \Gamma$, since $\omega_{\Lambda_{c}}^{\prime}=\omega_{\Lambda_{c}}$ and thus $\omega^{\prime}\left(P_{i}\right)=\omega\left(P_{i}\right)=1 \forall i \in \Lambda_{c}$. On the other hand, if $\Lambda_{1}, \Lambda_{2} \in L$ and $\omega^{\prime}\left(P_{\Lambda_{1}}\right)=\omega^{\prime}\left(P_{\Lambda_{2}}\right)=1$, then it follows from the relations $0 \leqq \omega^{\prime}\left(\left(I-P_{\Lambda_{1}}\right) P_{\Lambda_{2}}\right) \leqq \omega^{\prime}\left(I-P_{\Lambda_{1}}\right)=0 \quad$ that $\quad \omega^{\prime}\left(P_{\Lambda_{1} \cup \Lambda_{2}}\right) \equiv \omega^{\prime}\left(P_{\Lambda_{1}} P_{\Lambda_{2}}\right)=\omega^{\prime}\left(P_{\Lambda_{2}}\right)=1$. Hence, by induction, if $\Lambda_{1}, \Lambda_{2}, \ldots, \Lambda_{n} \in L$ and $\omega^{\prime}\left(P_{\Lambda_{1}}\right)=\omega^{\prime}\left(P_{\Lambda_{2}}\right)=\ldots=\omega^{\prime}\left(P_{\Lambda_{n}}\right)=1$, then $\omega^{\prime}\left(P_{\Lambda_{1} \cup \Lambda_{2} \ldots \cup \Lambda_{n}}\right)=1$. In view of this result, the assumption that $\omega^{\prime}\left(P_{i}\right)=1 \forall i \in \Lambda$, hence $\forall i \in \Gamma$, implies that $\omega^{\prime}\left(P_{\Lambda^{\prime}}\right)=1 \forall \Lambda^{\prime} \in L$, in contradiction with the assumption that $\omega^{\prime} \in \Omega \backslash \Omega_{0}$. Thus we have established, by a reductio ad absurdum argument, that $\exists i \in \Lambda$ for which $\omega^{\prime}\left(P_{i}\right)<1$. As a consequence, we see that, if $\Lambda^{\prime} \ni i$ and thus $P_{\Lambda^{\prime}}=P_{\Lambda^{\prime} \cup i}=P_{\Lambda^{\prime}} P_{i}$, then $\quad \omega^{\prime}\left(P_{i}\right)-\omega^{\prime}\left(P_{\Lambda^{\prime}}\right)=\omega^{\prime}\left(P_{i}\left(I-P_{\Lambda^{\prime}}\right)\right) \geqq 0$. Hence $1-\omega^{\prime}\left(P_{\Lambda^{\prime}}\right) \geqq 1-\omega^{\prime}\left(P_{i}\right)>0 \forall \Lambda^{\prime} \ni i$. It follows from this inequality and Equation (4), together with our specification of $\phi$, that

$$
\Delta \tilde{E}_{\Lambda}\left(\omega^{\prime} \mid \omega\right) \geqq-2\left|\phi_{1}\right|_{1}+\left(1-\omega^{\prime}\left(P_{i}\right)\right) \sum_{i \in \Lambda^{\prime}} c\left(\Lambda^{\prime}\right) .
$$

In view of the translational invariances of the forces, we may replace $\sum_{i \in \Lambda^{\prime}} c\left(\Lambda^{\prime}\right)$ by $\sum_{0 \in \Lambda^{\prime}} c\left(\Lambda^{\prime}\right)$ in this last estimate. Hence, as we are given that $\sum_{0 \in \Lambda^{\prime}} c\left(\Lambda^{\prime}\right)=\infty,\left|\phi_{1}\right|_{1}<\infty$ and $1-\omega^{\prime}\left(P_{i}\right)>0$, it follows that $\Delta \tilde{E}_{\Lambda}\left(\omega^{\prime} \mid \omega\right)=\infty$, as required.

Proof of (b). We shall prove (b) by showing that, for large enough $T, \inf \{f(\omega) \mid \omega \in \bar{\Omega}\}$ $<\inf \left\{f(\omega) \mid \omega \in \bar{\Omega}_{0}\right\}$.

For this purpose we note that it follows from equations (1), (3) that $\left|E_{\Lambda}(\omega) / N(\Lambda)\right| \leqq|\phi|$; while it follows from the definition of $S_{\Lambda}$ in [4(a)], together with our above specification of $\Omega, \Omega_{0}$ that $\sup \left\{\lim _{\Lambda \uparrow} S_{\Lambda}(\omega) / N(\Lambda) \mid \omega \in \bar{\Omega}\left(\operatorname{resp} . \bar{\Omega}_{0}\right)\right\}$

$=0(\operatorname{resp} . \log m / n)$. Hence, by Equation (5),

$$
\inf \{f(\omega) \mid \omega \in \bar{\Omega}\} \leqq|\phi|
$$

and

$\inf \left\{f(\omega) \mid \omega \in \bar{\Omega}_{0}\right\} \geqq-|\phi|+T \log n / m$.

Therefore $\inf \{f(\omega) \mid \omega \in \bar{\Omega}\}<\inf \left\{f(\omega) \mid \omega \in \bar{\Omega}_{0}\right\}$ for sufficiently large $T$, as required.

\section{References}

1. Sewell, G. L. : Ann. Phys. 97, 55 (1976)

2. Penrose,O., Lebowitz,J.L. : J. Stat. Phys. 3, 211 (1971)

3. Capocaccia,D., Cassandro,H., Olivieri,E.: Commun. math. Phys. 39, 185 (1974)

4. (a) Araki, H., Sewell, G. L.: Commun. math. Phys. 52, 103-109 (1977)

(b) Sewell, G.L.: Commun. math. Phys. 55, 53-61 (1977)

5. Ruelle,D.: Statistical mechanics. New York: Benjamin Inc. 1969

6. Emch,G.G. : J. Math. Phys. 8, 19 (1967)

Communicated by J. L. Lebowitz 